\title{
Influence of time-delayed feedback in the firing pattern of thermally sensitive neurons
}

\author{
M. Sainz-Trapága, ${ }^{1}$ C. Masoller, ${ }^{2}$ H. A. Braun, ${ }^{3}$ and M. T. Huber ${ }^{4}$ \\ ${ }^{1}$ Departamento de Física, Facultad de Ciencias Exactas y Naturales, Universidad de Buenos Aires, Buenos Aires, Argentina \\ ${ }^{2}$ Instituto de Física, Facultad de Ciencias, Universidad de la República, Igua 4225, Montevideo 11400, Uruguay \\ ${ }^{3}$ Institute of Physiology, Deutschhausstrasse 2, D-35037 Marburg, Germany \\ ${ }^{4}$ Department of Psychiatry, Deutschhausstrasse 2, D-35037 Marburg, Germany
}

(Received 6 February 2004; published 15 September 2004)

\begin{abstract}
We explore the dynamics of a Hodgkin-Huxley-type model for thermally sensitive neurons that exhibit intrinsic oscillatory activity. The model is modified to include a feedback loop that is represented by two parameters: the synaptic strength and the transmission delay time. We analyze the dynamics of the neuron depending on the temperature, the synaptic strength, and the delay time. We find parameter regions where the effect of the recurrent connexion is excitatory, inducing spikes or trains of spikes, and regions where it is inhibitory, reducing or eliminating completely the spiking behavior. We characterize the complex interplay of the intrinsic dynamics of the neuron with the recurrent feedback input and a noisy input.
\end{abstract}

DOI: 10.1103/PhysRevE.70.031904 PACS number(s): 87.19.La, 05.45.-a, 05.40.-a, 87.10.+e

\section{INTRODUCTION}

Many biological systems operate under the influence of time-delayed feedback mechanisms [1]. In particular, a single neuron might influence a recurrent loop though an auto-synapse, and/or though synaptic connections involving other neurons. Synaptic communication between neurons depends on propagation of action potentials along the axons. The finite conduction velocity and the information processing time in synapses lead to transmission delay times. The influence of such recurrent delayed loops on neuron response has been analyzed by several authors [2-11]. Two different approaches for modeling feedback loops have been considered. In models based on ordinary differential equations (ODE's), a term proportional to the membrane potential at an earlier time $\epsilon V(t-\tau)$ was added to the rate equation for the membrane potential, leading to a set of delay differential equations (DDE's). Here $\epsilon$ is the synaptic strength and $\tau$ is the delay time due to the sum of the conduction times along the axon and dendrites and processing times in other neurons. Assuming the Fitzhugh-Nagumo model, Plant [2] demonstrated that oscillations appeared for both, positive and negative feedback. In models based on finite difference equations, a recurrent excitatory (inhibitory) feedback loop has been implemented by increasing (decreasing) the membrane potential a certain amount $\epsilon$, at a time $\tau$ after each firing.

Diez-Martinez and Segundo [3] studied experimentally the pacemaker neuron in the crayfish stretch receptor organ and showed that as the transmission delay time was increased the discharge patterns went from periodic spikes to trains of spikes separated by silent intervals. They suggested that neuronal adaptation along the rapid successive firings played an important role in the observed behavior. The hypothesis was tested by Pakdaman et al. [4] using integrateand-fire and Hodgkin-Huxley models. They found that models with neuronal adaptation combined with recurrent excitation reproduced the experimental observations of Ref. [3]. Further analysis was done in Ref. [5], where the response to slow periodic modulation was investigated; in Ref.
[6], where the effects of noise and delay were investigated for both, a single neuron with a recurrent connection, and a fully interconnected neural network; and in Ref. [7], where the transfer function of a single neuron with recurrent connection, defined as the relationship between the constant input and the mean firing rate, was investigated.

The effect of delayed stimulation was also studied by Gomez et al. [8]. Through simulations of a compartmental Hodgkin-Huxley model they showed that for a pacemaker neuron that fires periodic spikes, recurrent excitation can induce to both an acceleration of the firings, leading to bursting patterns, or a slow down of the firings, leading to discharge patters where the interspike interval is several times longer than the interspike interval of the unperturbed neuron.

The effect of delayed stimulation has also been studied in the context of cardiology, where reentrant tachycardia has been experimentally studied using spontaneously bursting heart cells, stimulated with depolarizing current pulses delivered at a fixed delay time after each action potential [9]. Here the delay represents the reentrant path, the length of which could be varied by modifying the delay time. The complex patterns that arouse due to delayed stimulation were understood in terms of delicate interactions between phase resetting (which refers to the shifting of the timing of an oscillation as a consequence of its perturbation by an external stimulus) and overdrive suppression (which refers to the slowing of the frequency of an oscillation after rapid stimulation).

A fundamental feature of a time-delayed feedback loop is that it often leads to multistability. Based on integrate-andfire and Hodgkin-Huxley models, Foss et al. [10] found multistability in the form of qualitatively different neuron firing patterns, when the neuron is in a inhibitory feedback loop with appropriate choices of $\tau$ and $\epsilon$. Two kinds of multistability with respect to the initial function for the membrane potential were demonstrated, depending on whether the neuron was excitable or firing periodic spikes in the absence of feedback. Multistable systems can be used as memory storage devices, since information about different initial conditions is perpetuated in the form of different spike patterns. 
However, their potential use for memory storage is limited by the noise. The role of noise was later analyzed by Foss et al. [11], who showed that noise induced transitions between coexisting attractors. This indicated that noisy multistable systems are suited only for short-term memory storage because, in spite of the fact that the average time that a trajectory remains in a given attractor can be quite long, even for low noise there is a nonzero probability that a switch occurs to a different attractor. The experimental demonstration of multistability was later presented by Foss and Milton in Ref. [12]. A periodically spiking motoneuron of Aplysia Californica was reciprocally connected to a computer and the dynamics induced by the inhibitory loop was studied as a function of the time delay. It was observed that for certain choices of the delay several qualitatively different spike trains coexist. The observations were understood in terms of a mathematical model that incorporates the delay and the phase resetting properties of the neuron.

In the following we examine feedback effects and multistability in a model neuron that has been developed on the basis of experimental data from shark electroreceptors [13] and mammalian cold receptors [14]. The temporal sequence of spikes indicates that impulse generation of these neurons depends on subthreshold membrane potential oscillations. External stimuli alter the frequency and/or amplitude of the oscillations which can lead to pronounced changes of the impulse patterns. There are grouped discharges, so-called, bursts as well as single-spike discharges of different temporal structures. In electroreceptors and in the upper temperature range of cold receptors there is an irregular sequence of spikes which, however, show multimodal interval distributions indicating subthreshold oscillations which operates below but near the spike-triggering threshold. In this situation it essentially depends on noise whether a spike is triggered or not but the oscillation period is still reflected in the basic rhythm of the discharge. In contrast, irregular single-spike discharges which, in cold receptors, can be recorded at low temperatures do not have such distinct structure but seems to reflect pacemakerlike impulse generation with strong random fluctuations, eventually embedded in deterministic chaos.

A model was recently proposed by Braun et al. [15], which accounts for most observed features in these thermally sensitive neurons. This is a paradigmatic model of a most flexible neuronal pattern generator which can produce different types of impulse patterns that seem to be of relevance also in cortical neurons [16]. Therefore, it is most interesting to analyze what kind of effects can be obtained when such neurons are operating in specific dynamical states and are embedded in feedback loops. Here we propose the analysis of this model, extended to take into account a simple timedelayed autorecurrent connection. The model is a modification of the Hodgkin-Huxley model [17]. Two sets of depolarizing and repolarizing ionic conductances are responsible for spike generation and slow-wave potentials, respectively. As a function of the temperature different impulse patterns were identified [18], including coexistence of spikes and subthreshold oscillations (spikes with skippings), periodic (pacemaker) spikes, and bursting (spike-trains) patterns. This rich dynamic behavior is due to the interplay of the slow currents (which are absent in the original Hodgkin-Huxley model) and the spike-generating currents [19]. With addition of noise the model gives excellent agreement with experimental data of real biological neurons [20,21].

The model of Braun et al. has been employed by several authors to model sensory neurons that exhibit spontaneous intrinsic oscillatory activity. Neiman et al. [22] demonstrated experimentally that electroreceptor cells in the paddlefish contain an intrinsic oscillator that can be synchronized with an external signal, and numerical simulations based on Braun et al. model with a periodic external stimulus yield good agreement with the experimental observations. Feudel et al. [20] showed that there is a region of temperature values where an abrupt increase of the interspike intervals occurs. The origin of this unusual behavior was found to be in a homoclinic bifurcation of a saddle-focus fixed point embedded in a chaotic attractor. They also demonstrated that qualitatively the same phenomenon was observed in electrophysiological experiments with the caudal photoreceptor of the crayfish. Zhou and Kurths [23] studied the effect of common noise on the synchronization of neurons which are not coupled, based on the model of Braun et al. For identical neurons, complete synchronization was observed for large enough noise while for nonidentical neurons, phase synchronization was observed. It was also found that noise enhances synchronization of weakly coupled neurons. A saddle point embedded in the chaotic attractor was found to be responsible for these nontrivial noise-induced effects. Ciszak et al. [24] studied the synchronization of two neurons coupled unidirectionally (in a master-slave configuration) and under the influence of common noise, based on the model of Braun et $a l$. It was shown that under appropriate coupling conditions, the spikes fired by the slave neuron anticipate (i.e., predict) the spikes fired by the master neuron.

The aim of this paper is to analyze the effect of a timedelayed feedback loop on the firing patterns of the model of Braun et al. Our motivation is twofold. On one hand, recurrent feedback loops with fixed delay time are relevant to the study of hybrid systems that integrate real neurons with electronic devices through chemical or electrical synapses [25-29]. On the other hand, our motivation is to study a complex phenomenon that is relevant to nonlinear science: the interaction between excitatory dynamics and memory. In particular, our goal is to investigate the effect of a recurrent loop in the dynamics of subthreshold oscillations that operate close to but below that the spiking threshold. We have chosen to use the neuron model of Braun et al. because it is a most flexible pattern generator that, with the inclusion of noise, gives very good agreement with experimental data recorded from real neurons.

We show that for particular values of the delay time the recurrent loop modifies the amplitude of the subthreshold oscillations in a way that they operate slightly above threshold, therefore leading to feedback-induced spikes. To the best of our knowledge, this is a novel mechanism for the emergence of deterministic spike activity.

This paper is organized as follows. Section II presents a brief description of the model (full details can be found in Ref. [15]). Section III presents results of the numerical simulations. The temperature is varied to consider three different impulse patterns of the neuron without feedback: subthresh- 




FIG. 1. Effect of increasing the connection strength. $\tau=100 \mathrm{~ms}$, $T=35^{\circ} \mathrm{C}$, $\epsilon=$ (a) 0 , $\begin{array}{ll}\text { (b) } 0.01 \mathrm{~ms}^{-1}, & \text { (c) } 0.06 \mathrm{~ms}^{-1} \text {, }\end{array}$ (d) $0.07 \mathrm{~ms}^{-1}$, (e) $0.08 \mathrm{~ms}^{-1}$. old oscillations (in the presence of noise: spikes with skippings), periodic spikes, and spike trains. In each of these regimes we study the influence of the feedback loop and the effect of noise. Section IV presents a summary and our conclusions.

\section{MODEL}

The model is a modification of Braun et al. model for thermally sensitive neurons [15]:

$$
\dot{V}=\frac{1}{C_{M}}\left(-i_{\mathrm{Na}}-i_{\mathrm{K}}-i_{s d}-i_{s r}-i_{l}\right)+f[V(t-\tau)]+\sqrt{D} \xi(t),
$$

where $V$ is the potential voltage across the membrane, $C_{M}$ is the capacitance, $i_{\mathrm{Na}}(T)$ and $i_{\mathrm{K}}(T)$ are the fast sodium and potassium currents, $i_{s d}(T)$ and $i_{s r}(T)$ are additional slow currents. These four currents depend on the temperature $T$ as described in Ref. [15]. $i_{l}$ is a passive leak current. For details and definitions of the other quantities of the model of Braun et al. see Ref. [15].

The function $f[V(t-\tau)]$ in the second term of the righthand side (RHS) of Eq. (1) describes the effects of the recurrent feedback connection. $V(t-\tau)$ is the membrane potential at the earlier time $t-\tau$. As in Refs. [2,10] we consider a linear recurrent input which is proportional to delayed version of the neuron firing pattern: $f(V)=\epsilon\left(V-V_{0}\right)$. Here $\epsilon$ is the synaptic strength and $V_{0}$ is a reference value. The last term in the RHS of Eq. (1) is a Gaussian white noise that represents a random external stimulus, and $D$ is the noise strength.

\section{RESULTS}

The dynamics of the model in the absence of external stimulus and in the absence of a recurrent input depends on the temperature parameter, exhibiting qualitatively different firing patterns, such as subthreshold oscillations $\left(T=35^{\circ} \mathrm{C}\right)$, regular spikes $\left(T=7.2^{\circ} \mathrm{C}\right)$, and trains of spikes $\left(T=20^{\circ} \mathrm{C}\right)$ [18]. Here we study the effect of a recurrent connection in these different dynamic regimes. The parameters used are as in Ref. [18]. $V_{0}=-47 \mathrm{mV}, \epsilon$ and $\tau$ are free parameters. To integrate the equations we have to specify the value of the membrane potential $V(t)$ in the initial time interval $[-\tau, 0]$. It has been shown that different choices of $V(t)$ in the interval $[-\tau, 0]$ give rise to different firing patterns $[10,11]$. Here, the initial condition is taken all though the paper with the membrane potential equal to a constant negative value in the interval $[-\tau, 0]$.

The feedback loop introduces a rich and complex behaviour. In this paper we limit ourselves to study positive feedback $(\epsilon>0)$ with a delay time in the range $0<\tau<500 \mathrm{~ms}$. Since the conduction velocity along axons connecting neurons varies from 20 to $60 \mathrm{~m} / \mathrm{s}$, the transmission delay time is from milliseconds to hundreds of milliseconds, and taking into account that a neuron can receive back its own output after several synapses, the interval $0<\tau<500 \mathrm{~ms}$ is a range of biologically plausible delay times $[6,10]$.

As a first step we study the deterministic model $(D=0)$, and then assess the impact of Gaussian noise. Figure 1 displays the effect of increasing the synaptic strength $\epsilon$ when the temperature $T$ is such that without recurrent connexion the neuron exhibits subthreshold oscillations $\left(T=35^{\circ} \mathrm{C}\right)$. The delay time of the recurrent connexion is $\tau=100 \mathrm{~ms}$, of the same order as the period of the subthreshold oscillations $T_{S O}$ (without recurrent connection $T_{S O} \sim 133 \mathrm{~ms}$ ).

For comparison, Fig. 1(a) displays the subthreshold oscillations in the absence of recurrent connection $(\epsilon=0)$. For small $\epsilon$ there are no spikes, the membrane potential exhibits only subthreshold oscillations. As $\epsilon$ increases above a certain threshold $\epsilon_{\mathrm{th}}$ the recurrent connection has an excitatory effect since the neuron starts firing pulses periodically [Fig. 1(b)]. 




FIG. 2. Effect of increasing the connection. $\tau=20 \mathrm{~ms}, \quad T=35{ }^{\circ} \mathrm{C}, \quad \epsilon=$ (a) $0.006 \mathrm{~ms}^{-1}$,

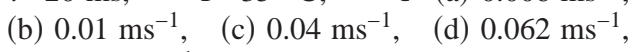
(e) $0.064 \mathrm{~ms}^{-1}$.

The value of $\epsilon_{\mathrm{th}}$ depends on the delay time, as will be discussed later $\left(\epsilon_{\mathrm{th}} \sim 0.002 \mathrm{~ms}^{-1}\right.$ for $\left.\tau=100 \mathrm{~ms}\right)$. As $\epsilon$ continues to increase, there is a gradual distortion of the spikes [Fig. 1(c)] and for larger $\epsilon$ the neuron fires one or two spikes per oscillating period [Fig. 1(d)]. We will refer to the firing of two spikes per oscillating period as two-spike trains. In Fig. 1(d) we observe a periodic sequence of two-spike trains alternating with one spike. For larger $\epsilon$ the neuron fires a periodic sequence of two-spike trains [Fig. 1(e)], such that the time interval between the last spike of a train and the first spike of the next train is $\sim \tau$. If the connection strength is increased further, the spiking behavior disappears and the membrane potential reaches a constant negative value (for $\left.\epsilon \sim 0.09 \mathrm{~ms}^{-1}\right)$. For even further increase of $\epsilon$ the membrane potential diverges (for $\epsilon \sim 0.1 \mathrm{~ms}^{-1}$ ).

The effect of the synaptic strength depends on the value of the transmission delay time. For larger $\tau\left(\tau>T_{S O}\right)$ the spiking dynamics is such that, for large enough $\epsilon$ the neuron fires trains of three or more spikes. On the contrary, for short $\tau\left(\tau \ll T_{S O}\right)$ we have not observed spike trains. As an example, Fig. 2 displays results for $\tau=20 \mathrm{~ms}$. For $\epsilon$ above a certain threshold $\epsilon_{\mathrm{th}}$ the neuron fires spikes, which occur in between one or more subthreshold oscillations [firings with skippings, see Figs. 2(a) and 2(b). A similar behavior has been observed in the context of the Belousov-Zhabotinsky reaction and has been studied in terms of asymmetric bimodal maps [30,31]. Following Ref. [30] we refer to the skipping behavior where there are $n$ oscillations between two consecutive spikes as $n$-peak spikes. As $\epsilon$ increases we observe the sequence: three-peak spikes [Fig. 2(a)], two-peak spikes [Fig. 2(b)], one-peak (single) spikes [Fig. 2(c)], twopeak spikes [Fig. 2(d)], and four-peak spikes [Fig. 2(e)].

The above results are summarized in Fig. 3 with the help of a bifurcation diagram, which is done by plotting the time interval between consecutive spikes (interspike interval) as a function of the connexion strength $\epsilon$. The regions of the diagram where no dots appear represent parameter regions where no spikes occur (for low $\epsilon$ the membrane potential exhibits only subthreshold oscillations, while for large $\epsilon$ either it reaches a constant negative value, or it diverges). For comparison, Fig. 3(a) displays the bifurcation diagram when there is no delay in the recurrent connection $(\tau=0)$, while Figs. 3(b) and 3(c) display the bifurcation diagrams corresponding to $\tau=20 \mathrm{~ms}$ (Fig. 2) and $\tau=100 \mathrm{~ms}$ (Fig. 1), respectively.

Without delay [Fig. 3(a)], it is observed that as $\epsilon$ increases the neuron starts firing spikes such that the time interval between consecutive spikes is two oscillation periods (i.e., two-peak spikes), then there is a large region of $\epsilon$ where the interspike interval is one oscillation period. Above a certain value $\left(\epsilon \sim 0.05 \mathrm{~ms}^{-1}\right)$ the neuron fires two-spike trains: there are two different values of the interspike interval, one small corresponding to the time interval between the two spikes in the train, and one large corresponding to the time interval between the last spike of a train and the first spike of the next train. The time interval between two consecutive trains increases with $\epsilon$. For $\epsilon$ large enough $\left(\epsilon>0.08 \mathrm{~ms}^{-1}\right)$ there are no spikes (either the membrane voltage reaches a constant value or diverges).

For a delay time of $\tau=20 \mathrm{~ms}$ [Fig. 3(b)] we observe the sequence of spikes as $\epsilon$ increases shown previously in Fig. 2: spikes separated by three oscillation periods (three-peak spikes), spikes separated by two oscillation periods (twopeak spikes), and so on. For a delay time of $\tau=100 \mathrm{~ms}$ [Fig. 3(c)] we observe a bifurcation diagram corresponding to the different spike patterns shown in Fig. 1: (i) for $0.002 \mathrm{~ms}^{-1}<\epsilon<0.066 \mathrm{~ms}^{-1}$ the neuron fires single spikes, (ii) for $0.066 \mathrm{~ms}^{-1}<\epsilon<0.07 \mathrm{~ms}^{-1}$ the neuron fires both, two-spike trains and single spikes (note that in this parameter region there are three points in the bifurcation diagram), (iii) for $0.07 \mathrm{~ms}^{-1}<\epsilon<0.09 \mathrm{~ms}^{-1}$ the neuron fires two-spike trains separated by a time interval $\sim \tau$.

In our simulations we observe multistability, i.e., the coexistence of different solutions for the same set of parameter values and different initial conditions. For example, for the parameters of Fig. 2(e) we observe depending on the initial 




FIG. 3. Interspike intervals vs the strength of the recurrent connection, for different delay times. $\quad T=35{ }^{\circ} \mathrm{C}, \tau=($ a) $0 \mathrm{~ms}$, (b) $20 \mathrm{~ms}$, (c) $100 \mathrm{~ms}$.

conditions either three-peak spikes, or the alternation of twopeak and three-peak spikes, or four-peak spikes. This agrees with the observation of multistability in Refs. [10-12]. In particular, the similarity with the results of Ref. [12] suggest that the neuron dynamics can be represented by the phase resetting curve (which relates the phase at which a perturbation arrives with the subsequent phase shift in the neuron spike train). This study is in progress and will be reported elsewhere.

We point out that the existence of a delay time in the recurrent connection modifies the neuron spiking behavior drastically, in two ways. As will be discussed below, there are intervals of delay time in which the recurrent connection does not induce any spiking behavior. For values of $\tau$ such that spikes occur, there is a type of locking behavior in the sense that as $\epsilon$ increases the interspike interval and the time interval between trains of spikes remain nearly constant or varies abruptly with $\epsilon$, while for $\tau=0$ it is observed that both the interspike interval and the time interval between trains increase smoothly with $\epsilon$ [Fig. 3(a)].

There are values of the delay time where the recurrent connection is inhibitory and values where it is excitatory. This can be observed in Fig. 4, where we show the interspike intervals versus $\tau$ for various values of $\epsilon$. For small $\epsilon$ [Fig. 4(a)] the regions where the recurrent connection is excitatory are centred at values of the delay time which are multiples of the subthreshold oscillations period: $\tau \sim n T_{S O}$ (the feedback loop modifies slightly the value of $T_{S O}$ with respect to the period of the oscillations in the absence of feedback: for $\epsilon$ $=0.002 \mathrm{~ms}^{-1} T_{S O}=125 \mathrm{~ms}$ ). In the regions where the feedback loop is excitatory the interspike interval is a multiple of $T_{S O}$. The fact that spikes occur for $\tau \sim n T_{S O}$ and that the interspike interval is also a multiple of $T_{S O}$ indicate that for weak synaptic strength the firing pattern is strongly influenced by the intrinsic subthreshold oscillatory behavior of the neuron without feedback.

The bifurcation diagram of Fig. 4(a) can be understood after a close inspection of the effect of the delay time on the subthreshold oscillations. We show in Fig. 5 the maximum, minimum and average values of the oscillations as a function of $\tau$, for various values of $\epsilon$. To distinguish simple periodic oscillations from more complex behaviors, we plot local maxima and minima for each oscillation cycle. For very small $\epsilon$ [Fig. 5(a)] no spikes occur for any $\tau$, and the effect of $\tau$ is to modify the amplitude of the subthreshold oscillations (which are close to but below the spike triggering threshold). For $\tau \sim n T_{S O}$ the oscillations are enhanced, approaching the threshold, while for $\tau \sim(n+1 / 2) T_{S O}$ they are diminished. For larger $\epsilon$ [Figs. 5(b)-5(d)] the effect of nonlinearities becomes more pronounced, leading to a larger distortion of the oscillations, and spikes occur in the regions of $\tau$ where the oscillations are enhanced. For even larger $\epsilon$ [Fig. 5(e)], the size of the regions of $\tau$ where spikes occur increases, and for values of $\tau$ where there are no spikes, the subthreshold oscillations

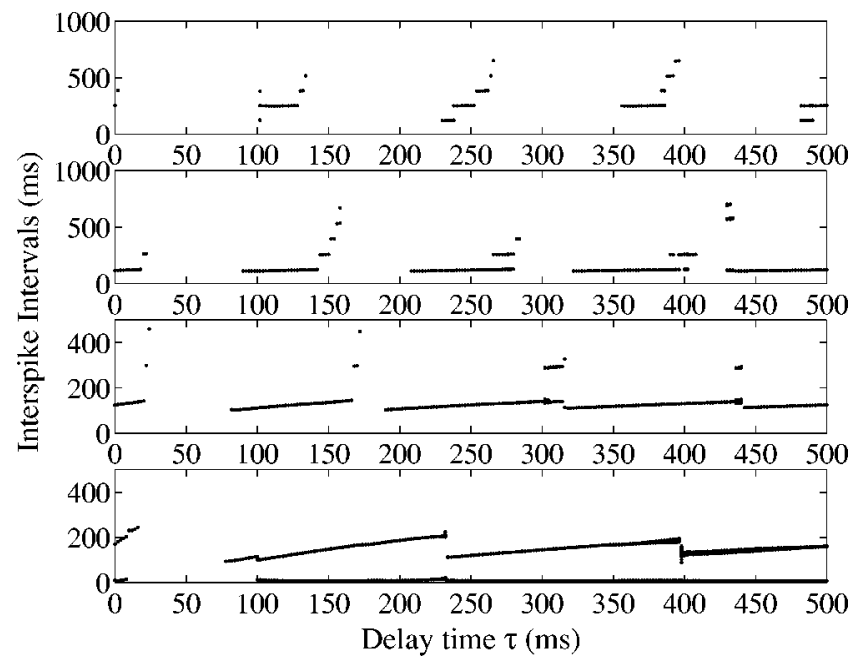

FIG. 4. Interspike intervals vs the time delay of the recurrent connection. $T=35{ }^{\circ} \mathrm{C}$, (a) $\epsilon=0.002 \mathrm{~ms}^{-1}$, (b) $\epsilon=0.01 \mathrm{~ms}^{-1}$, (c) $\epsilon$ $=0.03 \mathrm{~ms}^{-1}$, (d) $\epsilon=0.07 \mathrm{~ms}^{-1}$. 

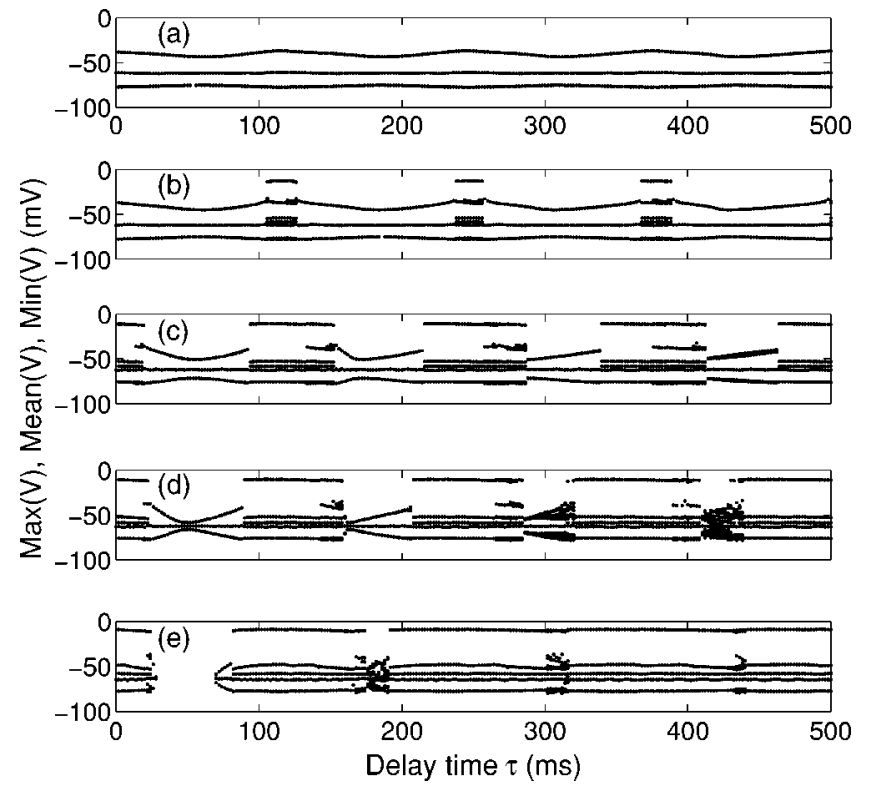

FIG. 5. Maximum, minimum, and average values of the oscillations of the membrane potential vs the transmission delay time. $T=35{ }^{\circ} \mathrm{C}$. (a) $\epsilon=0.001 \mathrm{~ms}^{-1}$, (b) $\epsilon=0.0015 \mathrm{~ms}^{-1}$, (c) $\epsilon$ $=0.005 \mathrm{~ms}^{-1}$, (d) $\epsilon=0.01 \mathrm{~ms}^{-1}$, (e) $\epsilon=0.03 \mathrm{~ms}^{-1}$.

become strongly distorted: they are either strongly dumped or they are quasiperiodic (with several local maxima and minima per oscillation cycle).

As the synaptic strength increases the regions of values of $\tau$ where spikes occur merge [Figs. 4(b) and 4(c)], and for large $\epsilon$ we show in Fig. 4(d) that the plot of the interspike intervals versus $\tau$ looks quite different. For small $\tau(\tau<20 \mathrm{~ms})$ and large $\tau(\tau>100 \mathrm{~ms})$ spike trains occur; for $20 \mathrm{~ms}<\tau<100 \mathrm{~ms}$ the recurrent connection is inhibitory and there are no spikes. For $\epsilon$ and $\tau$ large enough the time interval between trains is such that about $n$ trains occur for each delay time, with $n=1,2,3, \ldots$. In other words, for large $\epsilon$ and $\tau$ the time interval between trains goes as $\sim \tau$, $\sim \tau / 2, \sim \tau / 3, \ldots$. Typical spike trains for $\epsilon=0.08 \mathrm{~ms}^{-1}$ are shown in Fig. 6. The variation of the time interval between trains with the delay time indicates that for large synaptic strength the neuron firing pattern is strongly influenced by the recurrent connection with little influence of the intrinsic subthreshold oscillatory dynamics.

Let us now fix the temperature parameter such that the neuron fires periodic spikes. Figure 7(a) displays the spiking behavior for $T=7.2{ }^{\circ} \mathrm{C}$ without feedback. Two different interspike intervals can be observed (about $520 \mathrm{~ms}$ and 860 $\mathrm{ms})$. We first show results when the feedback loop has a small delay time $(\tau=20 \mathrm{~ms})$. As $\epsilon$ increases the difference between the interspike intervals gradually diminishes and for $\epsilon$ large enough the neuron fires spikes with a single interspike interval [Fig. 7(b)]. For $\epsilon$ above a threshold value the neuron fires spike trains [Fig. 7(c)]. The time interval between the train of spikes increases with $\epsilon$ [Fig. 7(d)], and for $\epsilon$ large enough the spikes disappear.

These results are summarized in the bifurcation diagram shown in Fig. 8(a), which resembles that of the neuron in the subthreshold oscillations regime [compare with Fig. 4(a); however, notice the difference in the vertical scale]. As before, the effect of the synaptic strength depends on the delay time of the recurrent connection. As an example, Figs. 8(b) and $8(\mathrm{c})$ display results for larger delay times $(\tau$ $=100$ and $300 \mathrm{~ms}$ ). A complex sequence of bifurcations for low $\epsilon$, and a locking regime for large $\epsilon$, where the neuron fires periodic spikes can be observed. For even larger $\epsilon$ the firings disappear and the membrane potential reaches a constant negative value.

For $\epsilon$ just below the onset of locking, intermittency occurs in the form of quiet periods interrupted by intense bursts of firing activity. Above the onset of locking, as $\epsilon$ increases the neuron gradually adapts its firing rate to fire an integer number of spikes in each $\tau$ time interval. For $\epsilon$ large enough Fig.



FIG. 6. Spike patterns for various delay times and large synaptic strength $\quad\left(\epsilon=0.08 \mathrm{~ms}^{-1}\right) . \quad T$ $=35^{\circ} \mathrm{C}$. (a) $\tau=200 \mathrm{~ms}$ : the time interval between spike trains is $\sim \tau$, (b) $\tau=300 \mathrm{~ms}$ : two spike trains occur for each $300 \mathrm{~ms}$, (c) $\tau=500 \mathrm{~ms}$ : three spike trains occur for each $500 \mathrm{~ms}$. 




FIG. 7. Effect of increasing the connection strength in the regular spikes regime. $\quad T=7.2^{\circ} \mathrm{C}, \tau$ $=20 \mathrm{~ms}$, $\epsilon=$ (a) $0.0 \mathrm{~ms}^{-1}$,
(b) $0.03 \mathrm{~ms}^{-1}$
(c) $0.06 \mathrm{~ms}^{-1}$,

(d) $0.074 \mathrm{~ms}^{-1}$.

9 shows that the interspike intervals are slightly larger than $\sim \tau / n$ with $n=1,2,3 \ldots$.

Next we study the effect of the feedback loop in a temperature region such that without recurrent connection the neuron fires trains of spikes. When there is no delay in the feedback loop, we observe in the plot of the interspike intervals vs $\epsilon$ [Fig. 10(a)] that several bifurcations occur and that the time interval between consecutive trains increases with $\epsilon$. While Fig. 10(a) clearly indicates the values of $\epsilon$ where bifurcations occur, it gives no information about the type of bifurcation that takes place, in the sense that an additional branch of interspike intervals for $\epsilon$ above a certain value might be due to the fact that the is an additional spike in the

train, or might be due to the fact that the interspike intervals change while the number of spikes in the train remains constant. A close inspection of the firing patterns shows that after each bifurcation the number of spikes in each train increases by 1 .

When the delay time is short (with respect to the time interval between consecutive trains) we observe that increasing $\epsilon$ has a similar effect [Fig. 10(b)]. On the contrary, for longer delay times the time interval between consecutive trains does not increase with $\epsilon$ but remains nearly constant [Fig. 10(c)] and a careful inspection of the firing patterns shows that the number of spikes in the trains also remains approximately constant with $\epsilon$ (there is, however, a disper-

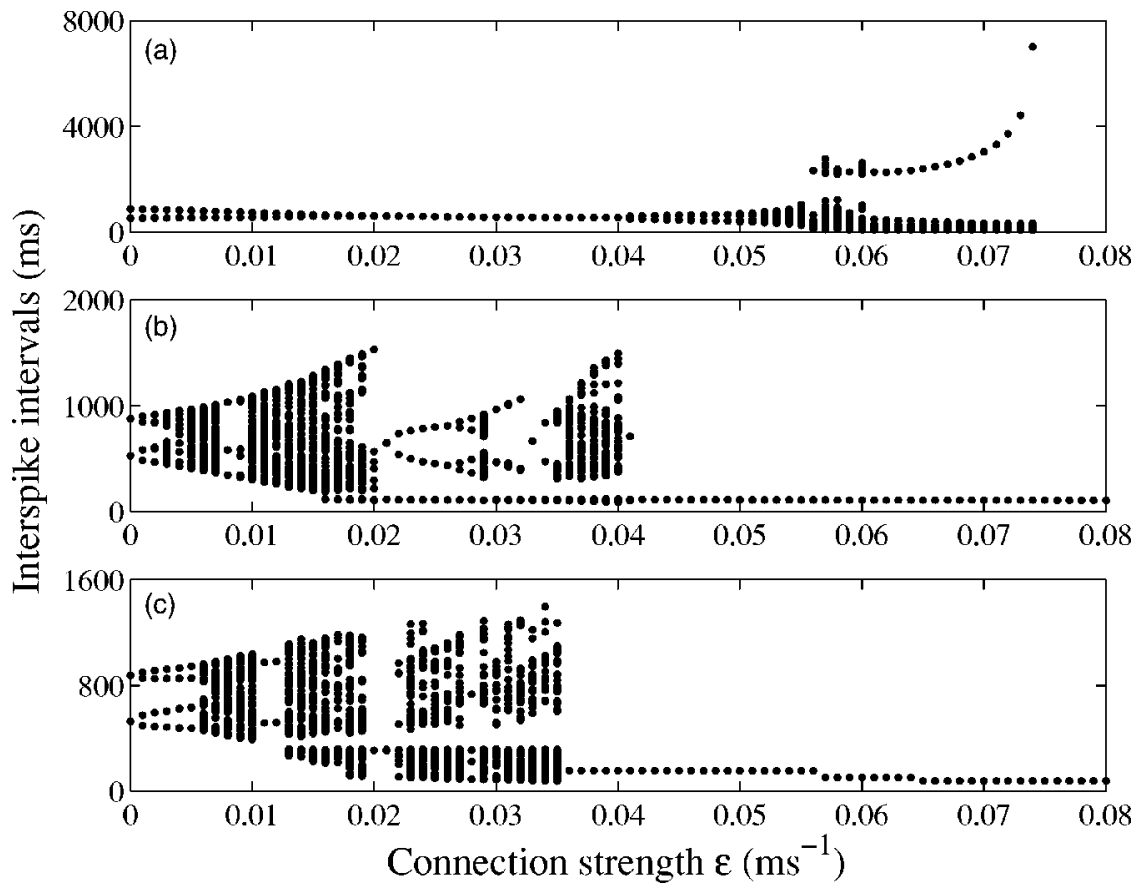

FIG. 8. Interspike intervals vs the strength of the recurrent connection, for different delay times. $T=7.2{ }^{\circ} \mathrm{C}, \quad \tau=$ (a) $20 \mathrm{~ms}$, (b) $100 \mathrm{~ms}$, (c) $300 \mathrm{~ms}$. 

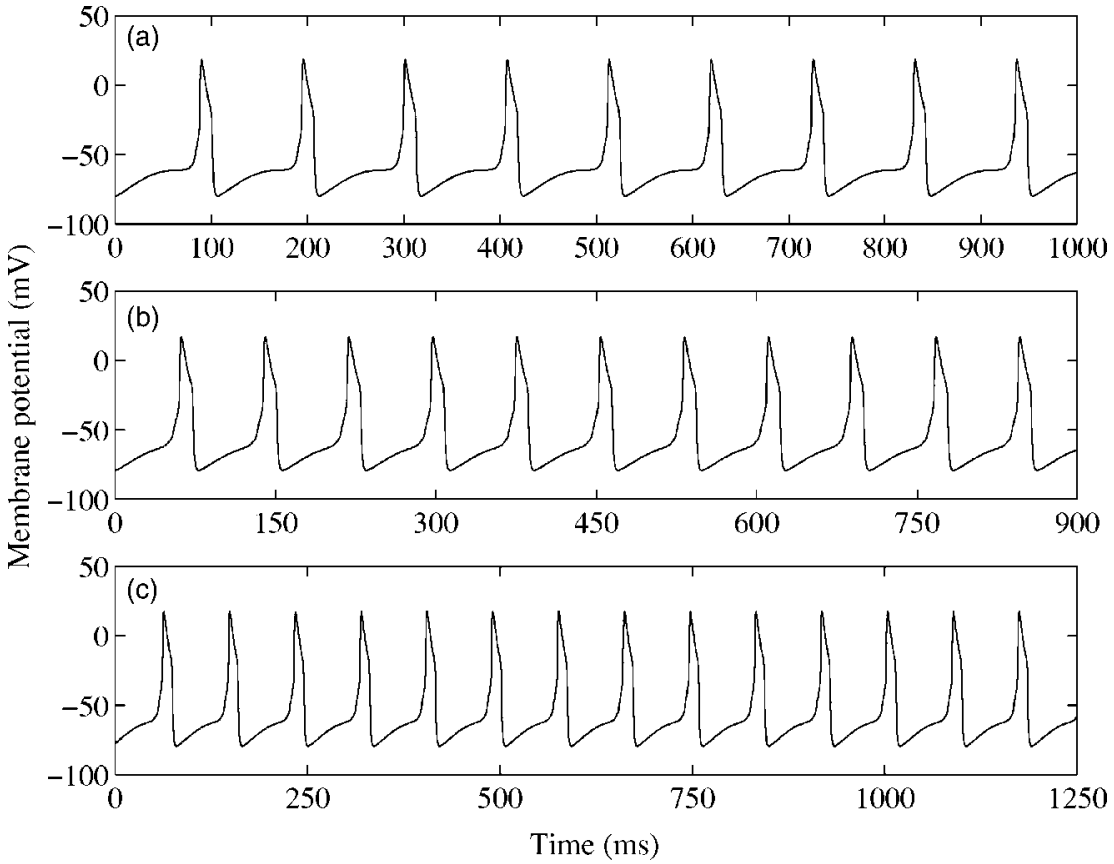

FIG. 9. Spike patterns for various delay times and large synaptic strength $\quad\left(\epsilon=0.08 \mathrm{~ms}^{-1}\right) . \quad T$ $=7.2{ }^{\circ} \mathrm{C}$. (a) $\tau=100 \mathrm{~ms}$ : the time interval between spikes is $\sim \tau$, (b) $\tau=150 \mathrm{~ms}$ : about two spikes occur for each $\tau$, (c) $\tau=250 \mathrm{~ms}$ : about three spikes occur for each $\tau$. sion of the interspike intervals for $\epsilon>0.04 \mathrm{~ms}^{-1}$ ). Increasing $\tau$ while keeping $\epsilon$ constant also leads to complex bifurcation diagrams where the number of spikes in the trains increases or decreases by one after each bifurcation. A detailed investigation of the influence of a feedback loop in the regime where the neuron fires spike trains is in progress and will be reported elsewhere.

Let us now assess the impact of noise, by including noise in the simulations. First we consider a temperature parameter such that without recurrent connection the membrane potential exhibits subthreshold oscillations $\left(T=35^{\circ} \mathrm{C}\right)$. Figures 11 and 12 display bifurcation diagrams for the same parameters as Figs. 3 and 4 but with a noise level $D=0.001 \mathrm{~ms}^{-1}$. In Fig. 11 we observe that for small $\epsilon$ the neuron is very sensitive to noise. Noise induces spikes such that the interspike interval is a random multiple of the subthreshold oscillation period (spikes with skippings). For large $\epsilon$ noise does not have a significant effect: the neuron fires spikes or trains of spikes whose time interval is about the same as in the deterministic case.

Comparing Figs. 4(a) and 12(a) we observe that for small $\epsilon$ noise also leads to an increase of the size of the windows of delay times in which spikes occur. The large dispersion of the values of the interspike intervals (which are multiples of $T_{S O}$ ) can also be observed. On the contrary, for large $\epsilon$ the neuron is much less sensitive to noise. Comparing Figs. 4(d) and $12(d)$ we observe that the effect of noise is only a small dispersion of the values of the interspike intervals.

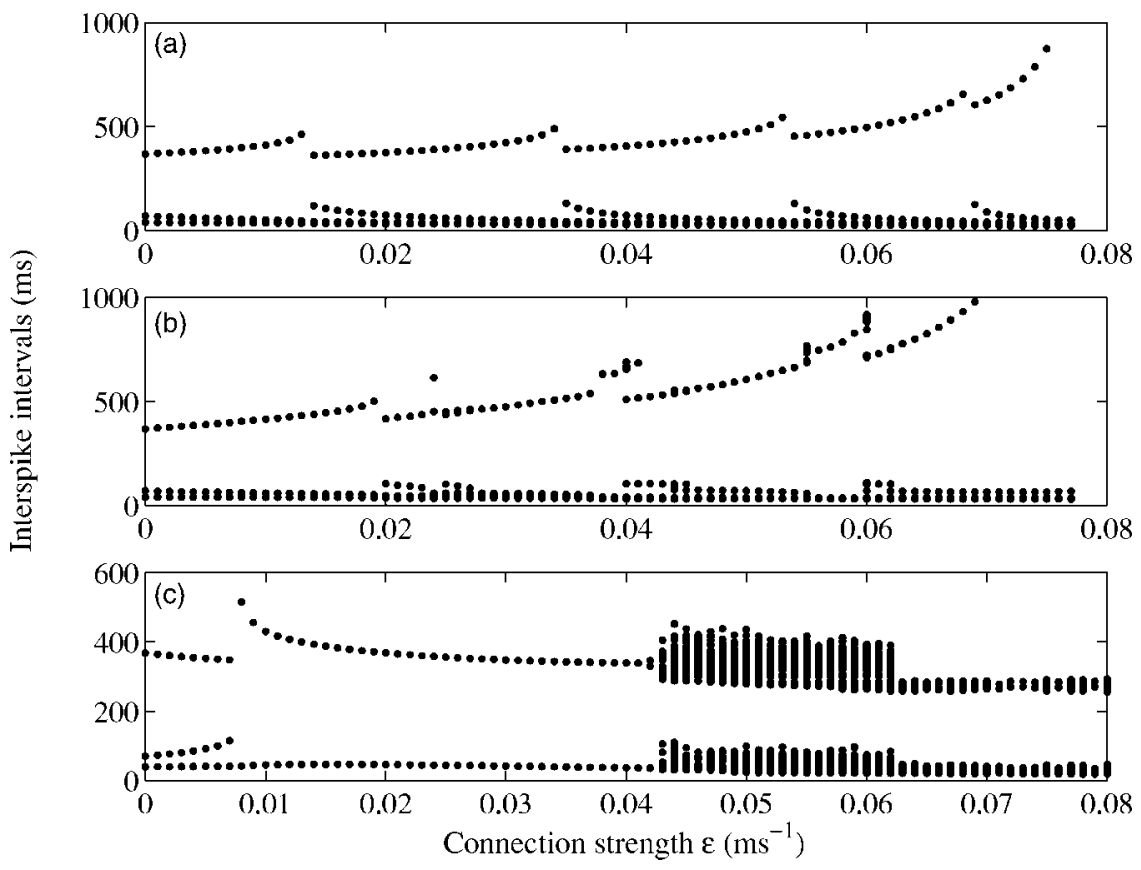

FIG. 10. Interspike intervals vs the strength of the recurrent connection, for various delay times. $\quad T=20^{\circ} \mathrm{C}, \quad \tau=$ (a) $0 \mathrm{~ms}$, (b) $100 \mathrm{~ms}$, (c) $300 \mathrm{~ms}$. 


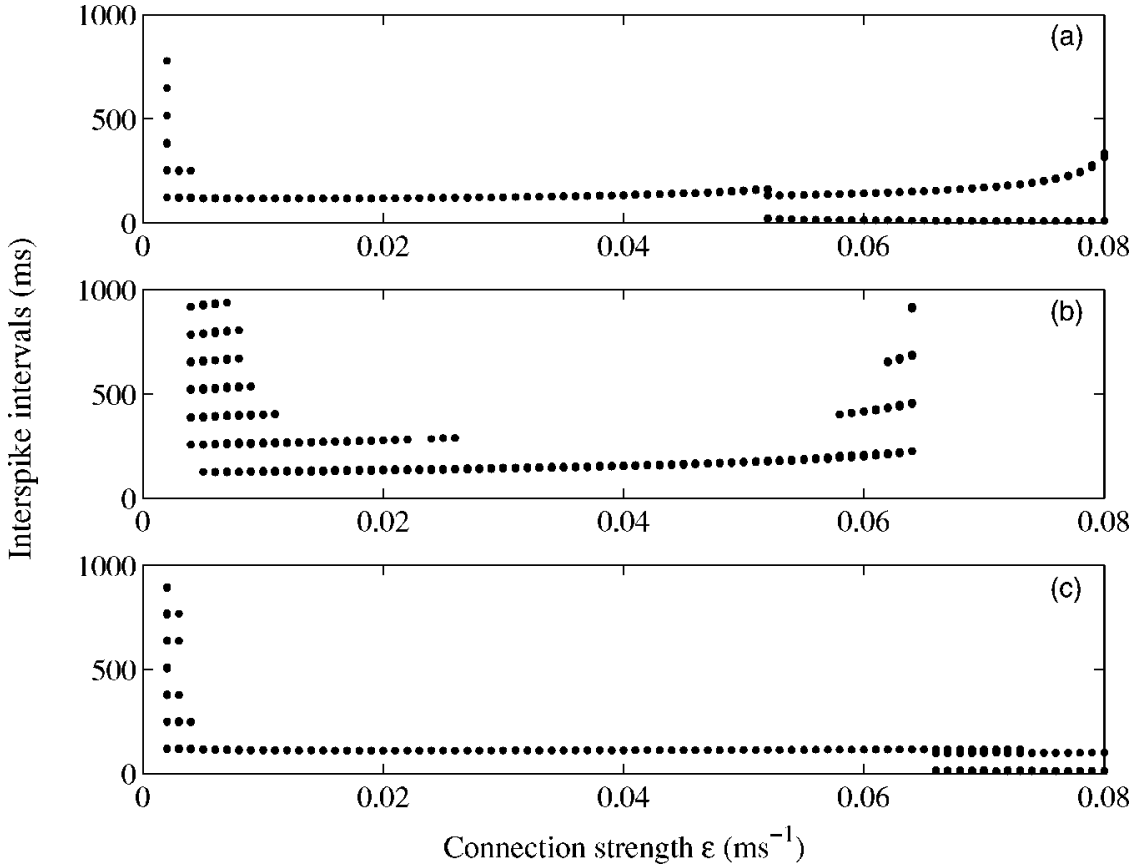

FIG. 11. Effect of noise in the subthreshold oscillations regime. Parameters are as in Fig. 3. D $=0.001 \mathrm{~ms}^{-1}$.
For temperatures such that without recurrent connection the neuron fires regular spikes or spike trains the effect of noise is to wash out the fine structure of the bifurcations occurring for low $\epsilon$. As an example, Fig. 13 shows results for $T=7.2{ }^{\circ} \mathrm{C}$. It can be observed that in the locking regime (for large $\epsilon$ and $\tau$ ) noise has a negligible effect.

\section{SUMMARY AND CONCLUSIONS}

In summary, we have studied the effect of a time-delayed recurrent connection on a model for thermally sensitive neurons that exhibit intrinsic oscillatory activity. The model is a Hodgkin-Huxley-type model that includes two sets of depolarizing and repolarizing ionic conductances which are responsible for spike generation and slow-wave potentials, respectively. The model describes temperature transduction in



FIG. 12. Effect of noise in the subthreshold oscillations regime. Parameters are as in Fig. 4. $D=0.001 \mathrm{~ms}^{-1}$. peripheral sensory receptors and as a function of the temperature parameter it exhibits different impulse patterns such as subthreshold oscillations, coexistence of spikes and subthreshold oscillations (spikes with skippings), and periodic (pacemaker) spikes and bursting (spike-trains) patterns.

We extended the model to include a feedback loop that was represented by two parameters: the synapse strength $\epsilon$ and the transmission delay time $\tau$. We studied the effect of the feedback loop in different temperature regimes.

In all temperatures regimes if the feedback is too strong the membrane potential is either constant or diverges. For large feedback we observed a locking of the neuron firing pattern with the delay time, such that the neuron fires single spikes or spike trains which are separated by time intervals related to the delay time. For weak feedback the effect of the feedback is more subtle and depends on the intrinsic dynamics of the neuron. Noise is found to have a strong influence on the dynamics for low feedback, where it leads to a large dispersion of the interspike intervals, but it does not play an essential role for large feedback.

While the effect of the feedback loop in the regular firing regime is similar to that found by other authors $[3,4,9,12]$, the observation that the feedback loop induces spikes in the regime of subthreshold oscillations of the isolated neuron has not, to the best of our knowledge, been reported before. We have understood this effect in terms of a perturbation of the amplitude of the subthreshold oscillations, which in the presence of feedback operate slightly above the firing threshold. It has been recently shown that the modification of the amplitude of an autonomous nonlinear oscillator (amplification or reduction depending on the feedback strength and the delay time) is a typical, generic, effect of a feedback loop [32].

In this paper we have used a type of linear feedback that is proportional to the difference between the membrane potential and a reference value. Naturally, this is a first approximation to the problem. From a physiological point of view the next step is to examine a feedback loop with different 


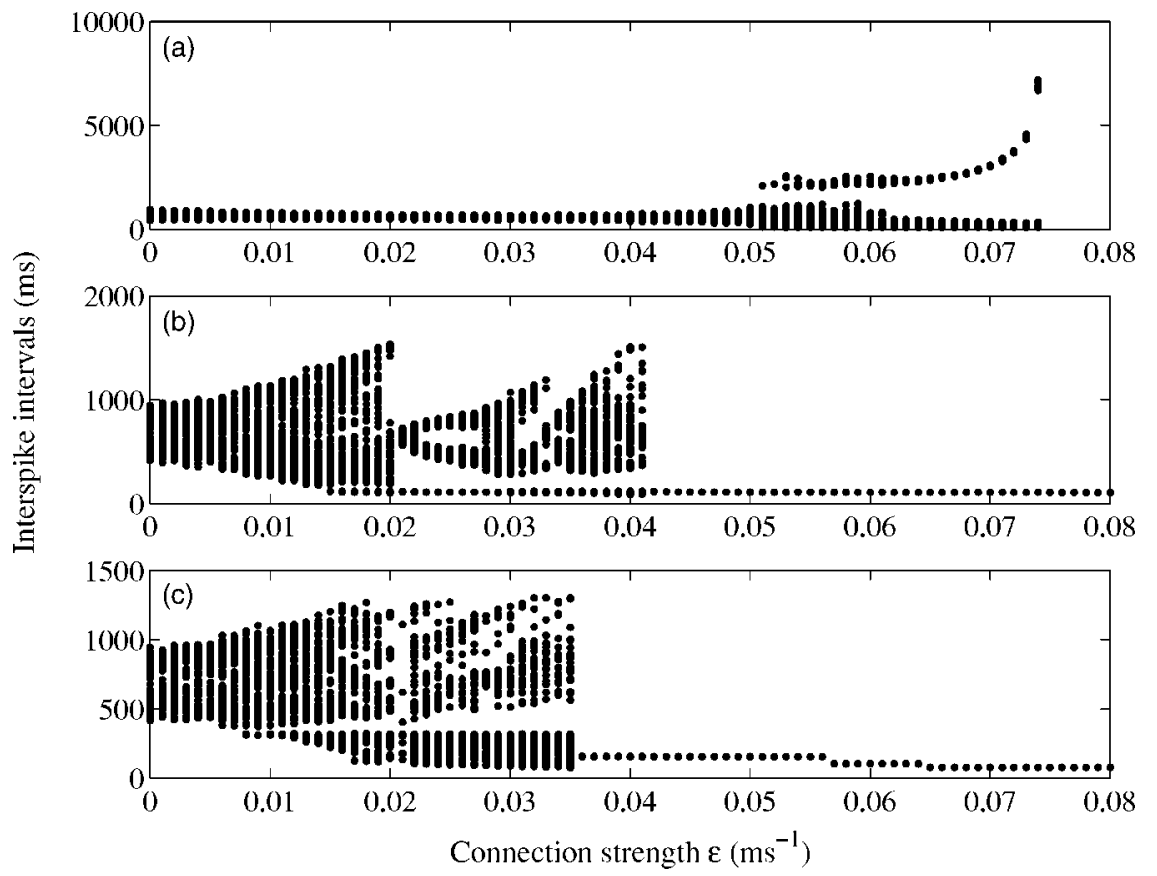

FIG. 13. Effect of noise. As in Fig. 8 but with $D=0.001 \mathrm{~ms}^{-1}$. nonlinearities that can depend on spike activity. As an example, it would be physiologically relevant to consider a global input from an ensemble of connected neurons, or the more specific effects of individual feedback neurons with a dynamic threshold for spike generation. The effect of the latter type of feedback is expected to be the same in the situation of subthreshold oscillations without spikes, but is expected that the feedback effects can considerably differ depending on whether the neuron is bursting, tonically firing, or is in the subthreshold oscillations regime firing occasional spikes due to noise. These studies are the object of future work.

\section{ACKNOWLEDGMENTS}

This work was partially carried out at the Abdus Salam International Centre for Theoretical Physics, Trieste, Italy. C.M. wishes to thank the Centre for hospitality.
[1] N. MacDonald, Biological Delay Systems: Linear Stability Theory (Cambridge University Press, Cambridge, 1989).

[2] R. E. Plant, SIAM (Soc. Ind. Appl. Math.) J. Appl. Math. 40, 150 (1981).

[3] O. Diez-Martines and J. P. Segundo, Biol. Cybern. 47, 33 (1983).

[4] K. Pakdaman, J. F. Vibert, E. Bousssard, and N. Azmy, Neural Networks 9, 797 (1996).

[5] K. Pakdaman, F. Alvarez, O. Diez-Martinez, and J. F. Vibert, BioSystems 40, 133 (1997).

[6] J. F. Vibert, F. Alvarez, and J. Pham, BioSystems 48, 255 (1998).

[7] K. Pakdaman, F. Alvarez, J. P. Segundo, O. Diez-Martinez, and J. F. Vibert, Int. J. Model. Simulat. 22, 260 (2002).

[8] L. Gomez, R. Budelli, and K. Pakdaman, Phys. Rev. E 64, 061910 (2001).

[9] A. M. Kunysz, A. Shrier, and L. Glass, Am. J. Physiol. 273, C331 (1997).

[10] J. Foss, A. Longtin, B. Mensour, and J. Milton, Phys. Rev. Lett. 76, 708 (1996).

[11] J. Foss, F. Moss, and J. Milton, Phys. Rev. E 55, 4536 (1997).

[12] J. Foss and J. Milton, J. Neurophysiol. 84, 975 (2000).

[13] H. A. Braun, H. Wissing, K. Schafer, and M. C. Hirsch, Nature
(London) 367, 270 (1994).

[14] H. A. Braun, H. Bade, and H. Hensel, Pfluegers Arch. 386, 1 (1980).

[15] H. A. Braun, M. T. Huber, M. Dewald, K. Schafer, and K. Voigt, Int. J. Bifurcation Chaos Appl. Sci. Eng. 8, 881 (1998).

[16] H. A. Braun, K. Voigt, and M. T. Huber, BioSystems 71, 39 (2003).

[17] A. L. Hodgkin and A. F. Huxley, J. Physiol. (London) 117, 500 (1952).

[18] W. Braun, B. Eckhard, H. A. Braun, and M. Huber, Phys. Rev. E 62, 6352 (2000).

[19] H. A. Braun, M. T. Huber, N. Anthes, K. Voigt, A. Neiman, X. Pei, and F. Moss, Neurocomputing 32, 51 (2000).

[20] U. Feudel, A. Neiman, X. Pei, W. Wojtenek, H. A. Braun, M. Huber, and F. Moss, Chaos 10, 231 (2000).

[21] H. A. Braun, K. Voight, J. C. Krieg, and M. T. Huber (unpublished).

[22] A. Neiman, X. Pei, D. Russell, W. Wojtenek, L. Wilkens, F. Moss, H. A. Braun, M. T. Huber, and K. Voigt, Phys. Rev. Lett. 82, 660 (1999).

[23] C. S. Zhou and J. Kurths, Chaos 13, 401 (2003).

[24] M. Ciszak, O. Calvo, C. Masoller, C. R. Mirasso, and R. Toral, Phys. Rev. Lett. 90, 204102 (2003). 
[25] Y. Yarom, Neuroscience 44, 263 (1991).

[26] P. Fromherz and A. Stett, Phys. Rev. Lett. 75, 1670 (1995).

[27] A. Stett, B. Müller, and P. Fromherz, Phys. Rev. E 55, 1779 (1997).

[28] R. C. Elson, A. I. Selverston, R. Huerta, N. F. Rulkov, M. I. Rabinovich, and H. D. I. Abarbanel, Phys. Rev. Lett. 81, 5692 (1998).
[29] R. A. Kaul, N. I. Syed, and P. Fromherz, Phys. Rev. Lett. 92, 038102 (2004).

[30] A. S. Pikovsky, Phys. Lett. 85A, 13 (1981).

[31] J. Losson, J. Milton, and M. C. Mackey, Physica D 81, 177 (1995).

[32] C. Masoller (unpublished). 